\title{
Das Oratorium im Spannungsfeld der Konfessionen: Zum interkonfessionellen Austausch von Oratorien im 18. Jahr- hundert
}

MARYAM HAIAWI, HAMBURG

\section{Einleitung}

Die Beziehung von Musik und Konfession im Oratorium des 18. Jahrhunderts gestaltete sich sehr spannungsreich. Zum einen war die Oratorienpraxis der damaligen unterschiedlichen Lokaltraditionen ein Teil der jeweiligen Konfessionskultur und diente der Vermittlung spezifischer konfessioneller Glaubensaussagen. Zum anderen war sie offen für interkonfessionellen Repertoireaustausch, was zeigt, dass ihre religiös-bekenntnishafte Dimension zuweilen von anderen Faktoren überlagert werden konnte und somit nicht immer das zentrale tragende Element darstellte.

Die Schwierigkeit, der Gattung „Oratorium“ einen festen Platz in den frühneuzeitlichen Konfessionskulturen zuzuweisen, ergibt sich folglich aus dem Umstand, dass die geistliche Dimension des Oratoriums je nach Kontext und Funktion unterschiedlich stark ausgeprägt sein konnte. Sie verband sich mit anderen Einflussfaktoren wie kulturpolitischen Motivationen, repräsentativen Bedürfnissen sowie dichtungs- und musikästhetischen Ansprüchen, welche die Oratoriendichtungen, -vertonungen und die Aufführungspraktiken wesentlich mitbestimmten. In der einschlägigen Gattungsforschung jedoch wurde die Frage nach der tatsächlichen Konfessionsgebundenheit oder -ungebundenheit des Oratoriums der Frühen Neuzeit und nach seiner Bewegung im Spannungsfeld der Konfessionen bislang nicht genauer untersucht. ${ }^{1}$ Insbesondere das durch interkonfessionellen Austausch entstandene Repertoire fand in diesem Kontext bisher wenig Beachtung, obwohl es das Gattungsverständnis im Hinblick auf das Verhältnis von Oratorium und Konfession wesentlich vertieft.

Die folgenden Ausführungen zielen daher darauf, die hier beschriebene Lücke der Gattungsforschung in den Vordergrund zu stellen und den Faktor "Konfession" im Oratorienrepertoire des 18. Jahrhunderts näher zu bestimmen. Zu diesem Zweck wird eine Auswahl von Werken besprochen, die eine interkonfessionelle Rezeption erfahren haben bzw. durch interkonfessionellen Austausch von Oratorienlibretti oder -vertonungen entstanden sind. Anhand ihrer

1 Arnold Schering, Geschichte des Oratoriums, Leipzig 1911. Howard E. Smither, A History of the Oratorio, 3 Bde., Chapel Hill 1977-1987. Günther Massenkeil, „Oratorium und Passion”, 2 Teile, in: Handbuch der musikalischen Gattungen, Bd. 10, hrsg. von Siegfried Mauser, Laaber 1998-1999. Irmgard Scheitler, Deutschsprachige Oratorienlibretti. Von den Anfängen bis 1730, Paderborn 2005. 
Werkbeschaffenheit und des an die jeweils neue Werkfassung gebundenen Aufführungskontexts wird nachvollziehbar, unter welchen Bedingungen überkonfessioneller Oratorientransfer möglich war, welche Impulse zum Repertoireaustausch führten und in welchen Bereichen textlich, musikalisch oder aufführungspraktisch - konfessionell bedingte Grenzen der Adaption lagen.

Der Ausgangspunkt der Überlegungen ist die grundlegende Beobachtung, dass das Verhältnis von Musik und Konfession nicht statisch, sondern dynamisch war. Als wortloses, zunächst "konfessionsneutrales" Medium wurde Musik in konfessionellen Aufführungskontexten mit spezifischen religiösen Bedeutungen aufgeladen. Dabei konnte die gleiche Musik aufgrund ihrer wandlungsfähigen Deutungsmöglichkeiten in verschiedenen konfessionellen Kontexten verwendet werden, d. h. Funktions- und Konfessionsgrenzen überschreiten. Hinzu kommt, dass sich Musik im Verlauf des 18. Jahrhunderts von semantischen Funktionen unabhängiger machte, sodass innermusikalische Aspekte neben Kontext- und Konfessionsfragen zunehmend an Bedeutung gewannen. Da sich das "Konfessionelle" nicht als feststehende Größe manifestierte, war es zudem möglich, dass sich das Oratorium in den Grenzbereichen von Liturgie und Konzert bzw. von geistlicher und weltlicher Musik bewegen konnte. Es besaß eine funktionale Wandlungsfähigkeit zwischen Andachtsmusik, höfischer Repräsentationsmusik, ästhetisch-religiösem Ereignis und konfessionellem Bekenntnis und variierte je nach Kontext zwischen theologischer Ausrichtung, Frömmigkeitspraxis, konfessionspolitischer Aussage oder Ästhetisierung konfessioneller Praktiken. Den geistesgeschichtlichen Hintergrund bildeten durch Einflüsse der Aufklärung bedingte theologische und frömmigkeitspraktische Transformationsprozesse und Umbrüche, die eine zunehmende Distanzierung von dogmatisch geprägten Glaubensanschauungen und eine Subjektivierung des religiösen Ausdrucks beförderten, ${ }^{2}$ was sich auch in den Inhalten und der musikalischen Gestaltung der Oratorien widerspiegelte.

\section{Interkonfessioneller Oratorienaustausch im 18. Jahrhundert: Übersicht über ausge- wählte Beispiele}

Die prägnantesten Beispiele zu konfessionsübergreifenden Adaptionen von Oratorienlibretti oder -vertonungen im 18. Jahrhundert stehen mit bedeutenden Musikzentren und Institutionen des deutschsprachigen Raums in Zusammenhang: Auf katholischer Seite geht es um Wien - den Wiener Kaiserhof und die dortigen städtischen Konzerte - und um den sächsischen Hof zu Dresden; auf protestantischer Seite handelt es sich um die lutherischen Städte Hamburg, Leipzig und Braunschweig. Allen Orten gemeinsam war ein im 18. Jahrhundert blühendes höfisches bzw. städtisches Musikleben, das im Bereich der Vokalmusik von den modernen italienischen Gattungen "Oper", "Kantate" und "Oratorium" getragen wurde. Die Oratorienaufführungen am Wiener und Dresdner Hof waren paraliturgische Veranstaltungen, jene in den

2 Vgl. Lothar Pikulkik, Leistungsethik contra Gefühlskult. Über das Verhältnis von Bürgerlichkeit und Empfindsamkeit in Deutschland, Göttingen 1984, S. 165. 
lutherischen Städten wurden vornehmlich im bürgerlichen Konzert, zuweilen auch im Gottesdienst dargeboten. Dazu trat im späteren 18. Jahrhundert ein weiterer Ort der Oratorienpflege, der in der Gattungsforschung aufgrund der Fokussierung auf die großen Traditionslinien bisher ganz vernachlässigt worden ist: die Herrnhuter Brüdergemeine, eine in den 1720er Jahren aus pietistischen Bewegungen hervorgegangene, im weiteren Sinn mit den vorreformatorischen Böhmischen Brüdern verbundene evangelische Freikirche. Die dortige Musikpraxis schöpfte in umfangreichem Maß aus protestantischem sowie katholischem Oratorienrepertoire, ${ }^{3}$ wobei in diesem Fall Kirchen- oder Konzertmusik ineinander übergingen, da das gesamte Musikleben der Herrnhuter von Religion und Glaube durchdrungen und davon geprägt war. ${ }^{4}$

In der Aufarbeitung von interkonfessionell genutzten Oratorien wird zwischen Werkfassungen, die durch Adaption einer fremdkonfessionellen Oratoriendichtung und solchen, die durch Übernahme einer Oratorienvertonung entstanden sind, unterschieden. Darüber hinaus liegt der Fokus auf Passionsoratorien bzw. Oratorienrepertoire der Passionszeit, da hauptsächlich die in diesem Kontext geschaffenen Werke im Interessenfeld des interkonfessionellen Austauschs standen, was u. a. mit den überkonfessionellen Gemeinsamkeiten in Bezug auf den Passionsstoff zu erklären ist.

Ein frühes Beispiel zur katholischen Rezeption eines protestantischen Oratoriums war Johann Ulrich von Königs Hamburger Passionsoratorium Die gekreuzigte Liebe ${ }^{5}$, das 1715 von Reinhard Keiser ${ }^{6}$ und 1731 von Georg Philipp Telemann für dortige Konzertaufführungen vertont wurde. ${ }^{7}$ Außerdem setzte es der Dresdner Hofkapellmeister Johann David Heinichen 1724 in stark gekürzter Form für das katholische Sepolcro am Dresdner Hof unter dem Titel Oratorio Tedesco in Musik. ${ }^{8}$ Schließlich fand Königs Dichtung in Auszügen Eingang in Andreas Weidners Sepolcro Die Beschuldigte Unschuld, das um 1733 von Georg Reutter d. J. für das Wiener Frauenkloster St. Jakob auf der Hülben vertont wurde. ${ }^{9}$

3 Die Ergebnisse basieren auf eigenen Quellenforschungen im Unitätsarchiv Herrnhut sowie in der Moravian Music Foundation Winston-Salem, NC.

4 Zur spezifischen Lebensform der Herrnhuter Brüdergemeine vgl. u. a. Dietrich Meyer, Zinzendorf und die Herrnhuter Brüdergemeine. 1700-2000, Göttingen 2009, S. 19-24.

5 Johann Ulrich von König, „Die gekreuzigte Liebe,/ Oder/ Thränen über das Leyden und Sterben unsers Heylandes, in einem Oratorio musicalisch aufgeführet", in: Des Herrn Königs Gedichte aus seinen von ihm selbst verbesserten Manuscripten gesammlet und herausgegeben, hrsg. von Johann Leonhard Rost, Dresden 1745, S. 579-594.

6 Dies lässt sich aus Keisers Soliloquiendruck von 1715 rekonstruieren: Reinhard Keiser, Seelige/ Erlösungs=Gedancken/ Aus dem ORATORIO/Der/ zum Tode verurtheilte und gecreuzigte/ JESUS, Hamburg 1715, Notenarchiv der Singakademie zu Berlin: SA 124 (2).

7 Georg Philipp Telemann, Die gekreuzigte Liebe oder Tränen über das Leiden und Sterben unseres Heilandes. Passionsoratorium nach den Worten von Johann Ulrich König. TWV 5:4, Textdruck, Zentrum für Telemann-Pflege und -Forschung, Magdeburg 2001. Unveröffentlicht.

8 Johann David Heinichen, Oratorio Tedesco al Sepolcro Santo. Partiturautograph (1724), Sächsische Landesbibliothek - Staats- und Universitätsbibliothek Dresden: Mus. 2389-D-63. Johann David Heinichen, Oratorio Tedesco al Sepolcro Santo, nach der autographen Partitur der Sächsischen Landesbibliothek - Staats- und Universitätsbibliothek Dresden: Mus. 2389-D-63. Manuskript, Depotarbeit für Das Erbe deutscher Musik, hrsg. von Hans Bergmann, 1989.

9 Andreas Weidner, Die Beschuldigte Unschuld. Und Der zum Todt des Kreuzes Verurtheilte Welt-Erlöser, Wienbibliothek: A 5491. 
Weitreichender war die protestantische Rezeption der Oratorien des Wiener Hofpoeten Pietro Metastasio, der konfessionsübergreifend als Opernlibrettist geschätzt wurde. Dies lässt sich beispielhaft anhand der Vertonungen seiner Passionsdichtung La Passione di Gesù Cristo erkennen. Neben der Erstvertonung durch Antonio Caldara für das Sepolcro am Wiener Kaiserhof 1730 sind zwei Wiederverwendungen der katholischen Passionsdichtung für protestantische Zwecke zu beobachten, welche die Formen der interkonfessionellen Rezeption und Transformation sehr anschaulich machen: 1751 vertonte der Thomaskantor Gottlob Harrer eine deutsche Übersetzung für die Karfreitagsvesper in der Nikolaikirche ${ }^{10}$ und 1763 entstand durch Jacob Schuback eine Neuvertonung der italienischen Originalfassung für das Hamburger Konzert "Auf dem Kamp".11

In Bezug auf Neutextierungen von Oratorien gingen die umfangreichsten Austauschbewegungen von Johann Adolph Hasses Karwochenoratorien für den katholischen Hof zu Dresden aus. Diese prägten - ähnlich wie seine Opern - die europäische Musikkultur des 18. Jahrhunderts konfessionsübergreifend und fanden auch in der Herrnhuter Brüdergemeine Eingang. Am deutlichsten lässt sich der Werktransfer an seinem Oratorium I Pellegrini al Sepolcro di Nostro Signore nach der Dichtung des Dresdner Hofpoeten Stefano Benedetto Pallavicino erkennen, das 1742 am Dresdner Hof uraufgeführt worden war. ${ }^{12}$ Hauptsächlich in Leipzig und Braunschweig wurde es vielfältig wiederverwendet. So fand neben Aufführungen im Leipziger Großen Concert ${ }^{13}$ 1767 eine Konzertdarbietung von Hasses Musik mit einer Neutextierung durch Johann Samuel Enderlein (Betrachtung des Leidens Jesu) statt. ${ }^{14}$ Diese Fassung wurde auch für eine Aufführung in den Leipziger Hauptkirchen genutzt und erhielt zudem Eingang in Braunschweig. ${ }^{15}$ Dort

10 Gottlob Harrer, Passions Cantate, Stimmensatz, Biblioteka Gdańska Polskiej Akademii Nauk: Ms Joh.216. Gottlob Harrer, La Passione del Nostro Signore, HarWV 50, hrsg. von Christoph Koop, Leipzig 2013. Unveröffentlicht. Ich danke Christoph Koop für die Überlassung der Transkription.

11 Jacob Schuback, La Passione di Gesù Cristo Signor Nostro, Staatsbibliothek zu Berlin - Preußischer Kulturbesitz: Mus. ms. 20212.

12 Johann Adolph Hasse, I Pellegrini al Sepolcro di Nostro Signore, Partiturabschrift der Österreichischen Nationalbibliothek: Mus.Hs.18150. Johann Adolph Hasse, I Pellegrini al Sepolcro di Nostro Signore. Oratorio in due parti, hrsg. von Gaetano Pitarresi, Bologna 2017.

13 In den 1750er Jahren wurde I Pellegrini gleich dreimal aufgeführt: am 23. März 1750, am 27. März 1752 sowie am 4. April 1757. Zur Aufführung 1750 gibt es ein erhaltenes Exemplar im Besitz der Königlichen Bibliothek Belgien: Fétis 4547 A 1 Mus. Das Textbuch zur Aufführung 1752 kann durch einen bis 1927 existierenden Standortkatalog der Sächsischen Landesbibliothek - Staats- und Universitätsbibliothek rekonstruiert werden: Olim Lit. Ital. D 1406. Vgl. Manuel Bärwald, Italienische Oper in Leipzig (1744-1756), Teilband 2: Katalogteil, Beeskow 2016, S. 515. Das Textbuch zur Wiederaufführung 1757 ist nicht erhalten. Vgl. Hermann von Hase, „Breitkopfsche Textdrucke zu Leipziger Musikaufführungen zu Bachs Zeiten", in: Bach-Jahrbuch 10 (1913), S. $110 \mathrm{ff}$.

14 Johann Samuel Enderlein, Betrachtung des Leidens Jesu: eine Cantate, nach der hassischen Composition des italiänischen Oratorii: I PELLIGRINI AL SEPOLCRO DI NOSTRO SALVATORE, Textdruck, Leipzig 1767, Bach-Archiv Leipzig: Rara II, 169-D.

15 Johann Adolph Hasse, Herrn Johann Adolph Haßens Churfürstlich Sächsischer Oberkapellmeister Music zu dem Italiänischen Oratorio Gli Pelligrini mit deutscher Poesie zu einer Paßions Music von J. S. Enderlein und eingeschalteten Chorälen, unterlegt von Herrn Johann Friedrich Doles, Cantore und Collega der Schule zu S. Thomas in Leipzig, Library of Congress (Music Division), Washington D. C.: M 2000. H 31 P 45, 1750 z. Johann Adolph Hasse, Oratorium zur Betrachtung des Leidens Christi. Die Musik von Herrn Johann Adolph Hassen. Königl. Kapellmeister, M 2000. H 31 32, Case. 
erfuhr I Pellegrini eine noch weitreichendere Rezeption: Zum einen schuf der Braunschweiger Dichter Justus Friedrich Wilhelm Zachariä auf der Grundlage von Pallavicinos Dichtung eine Neufassung (Die Pilgrime auf Golgatha), ${ }^{16}$ die u. a. von Johann Christoph Friedrich Bach am Bückeburger Hof (ca. 1769) ${ }^{17}$ und von Johann Georg Albrechtsberger für die Wiener TonkünstlerSocietät (1781) vertont wurde. ${ }^{18}$ Zum anderen übersetzte der Braunschweiger Literat Johann Joachim Eschenburg Pallavicinos Dichtung (Die Pilgrimme beym heiligen Grabe) für eine Aufführung mit Hasses Musik am dortigen Bildungsinstitut Collegium Carolinum (1771). ${ }^{19}$

Die Rezeption von Hasses Oratorien in der Herrnhuter Brüdergemeine unterschied sich von allen anderen Aneignungsformen: Erstens wurden einzelne Sätze selektiert und zweitens Text und Musik erheblich bearbeitet, um den spezifischen, an das besondere Musikverständnis der Herrnhuter gebundenen Anforderungen gerecht zu werden. Diese resultierten aus der von pietistischen Strömungen herrührenden Idee, Musik zum unmittelbaren Ausdruck des Glaubensgefühls zu machen und sie vorrangig in ihrem andachtspsychologischen Wert zu begreifen. ${ }^{20}$

\section{Beispiel 1: Rezeption von Königs Die gekreuzigte Liebe in Dresden und Wien}

Königs Passionsoratorium Die gekreuzigte Liebe (1715), das auf der Frühfassung Thränen unter dem Creutze Jesu (1711) basiert, ${ }^{21}$ zeichnet sich dadurch aus, dass lutherische Glaubensinhalte, die den Bekenntnischarakter des Oratoriums stärken, mit affektiven Darstellungen verbunden sind, die sowohl im Dienst der Passionsbetrachtung als auch der opernhaften Dramaturgie des Oratoriums stehen. Telemanns Vertonung für das Hamburger Konzert stellt ein aussagekräftiges Beispiel dafür dar, wie in der Etablierungsphase des protestantischen Konzertoratoriums im frühen bis mittleren 18. Jahrhundert konfessionelle oder religiöse und ästhetische Ansprüche im Rahmen der außerkirchlichen Aufführung zu einer Synthese geführt wurden. Kirchenmusikalische Bezüge werden im Kompositionsstil der Chöre (Turba- und polyphone Chöre) sowie

16 Justus Friedrich Wilhelm Zachariae, Die Pilgrime auf Golgatha, in: Ders., Poetische Schriften. Zweyter Theil (Sammlung der besten deutschen prosaischen Schriftsteller und Dichter. Vier und sechzigster Theil), Karlsruhe 1777, S. 467-483.

17 Johann Christoph Friedrich Bach, Die Pilgrime auf Golgatha, hrsg. von Ulrich Leisinger. Stuttgart 1997.

18 Johann Georg Albrechtsberger, Die Pilgrime auf Golgatha, Partitur, Ungarische Nationalbibliothek Széchényi Budapest: Ms. mus. 2.596.

19 Johann Joachim Eschenburg, „Die Pilgrimme beym heiligen Grabe. Ein Oratorium nach der haßischen Musik der Pellegrini", in: Hamburger Unterhaltungen 10 (1770), H. 4, S. 267-279. Johann Adolph Hasse, L'Oratorio I Pellegrini. Del Sign. Giovanni Adolphi Hasse, Herzog-August-Bibliothek Wolfenbüttel: 111 Mus. Hdschr.

20 Vgl. Philipp Jacob Spener, Erste Geistliche Schriften, 2. Teil, Frankfurt 1699, S. 22. Anja Wehrend, Musikanschauung, Musikpraxis, Kantatenkompositionen in der Herrnhuter Brüdergemeine. Ihre musikalische und theologische Bedeutung für das Gemeinleben von 1727 bis 1760, Frankfurt a.M. 1995, S. 99.

21 Johann Ulrich von König, Thränen unter dem Creutze Jesu./In einem Oratorio, Montags/ Dienstags und Mittwochs zur Vesper-Zeit in der Stillen Woche Musicalisch aufgeführet, Hamburg 1711, Hamburger Staatsarchiv: H46: Sammelband Nr. 217, Acta Hamb. Tom. XVII (1711-12), Nr. 16. Johann Ulrich von König, "Thränen unter dem Creutze Jesu [....]", in: Theatralische/ geistliche/ vermischte und Galante Gedichte/ Allen Kennern und Liebhabern der edlen Poesie/ zur Belustigung/ ans Licht gestellt, hrsg. von Johann Ulrich von König, Leipzig und Hamburg 1713, S. 307-323. 
im Einbezug von Choralstrophen erkennbar; Königs spezifische Fokussierung auf das Moment der Betrachtung und Verinnerlichung des Passionsgeschehens übersetzt Telemann mit einer äußerst nuancierten Klangfarbengestaltung sowie einer oberstimmenbetonten Schreibweise, wobei tendenziell weniger Affekte der Erschütterung, sondern vielmehr der "Rührung" angestrebt werden. Zudem weisen einige opernhafte Ariensätze, die primär auf die Theatralik der Szene abheben, auf die spezifische Verbindung von Unterhaltung, ästhetischem Genuss und inhaltlicher Botschaft im Konzertkontext.

Heinichens Vertonung von Königs gekürzter Oratorienfassung markierte den Beginn einer katholischen Oratorienkultur des Dresdner Hofes, die ihren Höhepunkt im Schaffen von Johann Adolph Hasse hatte. Die interkonfessionelle Rezeption von Königs Libretto in Dresden entstand durch den Textdichter selbst, der seit 1721 Hofpoet des sächsischen Kurfürsten und polnischen Königs Friedrich Augusts I. war. ${ }^{22}$ Die Bearbeitung für den spezifisch katholischen Aufführungskontext des Dresdner Sepolcros, das heißt einer Musik im Rahmen der Passionsandacht vor dem Heilig-Grab-Aufbau, hatte ganz offensichtlich Versuchscharakter, da sich weder aus der Art der Textkürzung noch der Vertonung ein Konzept erkennen lässt, das konsequent auf den konfessionellen oder höfischen Kontext abgestimmt war. Die textliche Bearbeitung und Neuvertonung spiegelten zu einem gewissen Teil inhaltliche und stilistische Unsicherheiten in Bezug auf das Sepolcro sowie praktische Unzulänglichkeiten wie etwa mangelndes Sängerpersonal wider. Abgesehen von einigen praktischen Angaben zu Länge und Grundcharakter der Darbietung - ein Sepolcro war traditionell einteilig und aufgrund des Passionskontextes in gemäßigter Virtuosität gehalten ${ }^{23}$ - bestanden offenbar keine festen Rahmenbedingungen in Bezug auf die konfessionelle Ausrichtung der Aufführung oder librettistische und musikalische Vorbilder, sodass Dichter und Komponist über relativ große Gestaltungsfreiheiten verfügten. So ist es zu erklären, dass die Textselektion nicht nach eindeutig "katholischen" Kriterien in Bezug auf die dichterische Aufbereitung der Passion erfolgte - die Marienklage wurde zum Beispiel gestrichen - und die Vertonung primär an den Geschmackskonventionen Friedrich Augusts II. und Heinichens eigenen, galanten und dem Opernstil entstammenden Kompositionsprämissen orientiert war und weniger an einem höfisch-konventionalisierten kirchenmusikalischen Stil wie etwa in Wien. Das bedeutet, dass der interkonfessionelle Transfer zwischen Hamburg und Dresden, der sich über Königs Oratoriendichtung ereignete, in erster Linie nicht mit konfessionellen Erwägungen verbunden, sondern vielmehr pragmatisch motiviert war. In der Aufbauphase einer katholischen Kirchenmusik bediente man sich einer geistlichen Dichtung

22 Max Rosenmüller, Johann Ulrich von König. Ein Beitrag zur Literaturgeschichte des 18. Jahrhunderts, Leipzig 1896, S. 21ff. Peter Heßelmann, Art. "König, Johann Ulrich", in: Killy Literaturlexikon. Autoren und Werke des deutschsprachigen Kulturraumes, 2., vollständig überarbeitete Auflage, Bd. 6, hrsg. von Wilhelm Kühlmann, Berlin/New York 2009, S. 553-555. Steffen Voss (Hellmuth Christian Wolff), Art. "König, Johann Ulrich von”, in: Die Musik in Geschichte und Gegenwart, 2., neubearb. Ausgabe, Personenteil Bd. 10, hrsg. von Ludwig Finscher, Kassel etc. 2003, Sp. 493-496.

23 Vgl. Howard E. Smither, A History of the Oratorio, Bd. 1: The Oratorio in the Baroque Era. Italy, Vienna, Paris, Chapel Hill 1977, S. 381. 
des Hofpoeten, dessen Anliegen es weiterhin gewesen sein könnte, die Pflege der deutschen Sprache, für die er sich allgemein einsetzte, auch in der Dresdner Hofkultur zu fördern. ${ }^{24}$ Heinichens Musik orientierte sich in keiner Weise an konfessionalisierten Formen der Passionsmusik, etwa den musikalischen Praktiken des Wiener Hofes, die sich als Ausdruck der spezifischen katholischen Frömmigkeit des Habsburgerhauses, der Pietas Austriaca, verstanden. ${ }^{25}$

Eine weitere Rezeption von Königs Passionsoratorium, die der Forschung bisher unbekannt war, ereignete sich im klösterlichen Kontext in Wien. Weidners Dichtung Die Beschuldigte Unschuld enthält umfangreiche Partien aus Königs Thränen unter dem Creutze Jesu sowie Die gekreuzigte Liebe. Dabei handelt es sich um jene Passagen aus Königs Oratorium, die vom Charakter her betrachtend angelegt sind und das Kreuzigungsgeschehen reflektieren. Weidners Textzusammenstellung ist dabei vorrangig pragmatischer Natur, wie die uneinheitliche Stilistik seiner eigenen Verse und denen Königs zeigt. Der Fokus auf die betrachtenden Teile könnte aber darauf hinweisen, dass dieses Moment der Königschen Dichtung in idealer Weise dem Wiener klösterlichen Sepolcro entsprach. Im Dresdner Oratorio Tedesco wiederum war in Bezug auf die Textselektion keine so klare inhaltliche Schwerpunktsetzung erkennbar. Auf welchem Weg Königs Libretti nach Wien gekommen sind, ist nicht erforscht. Naheliegend ist der Transfer über Dresden, wobei Weidner sich aber auf die Hamburger Fassungen des Passionsoratoriums und nicht auf die gekürzte Textfassung aus Dresden bezog.

\section{Beispiel 2: Rezeption von Metastasios La Passione in Leipzig und Hamburg}

Das Besondere von Metastasios Dichtung La Passione besteht darin, dass in ihr zeitgenössische Reformprinzipien und durch den Wiener Aufführungskontext bedingte Anforderungen miteinander verbunden sind: Auf dichtungsästhetischer Ebene verfolgte Metastasio das Konzept, das Passionsoratorium als Drama zu gestalten; in Bezug auf die Konventionen des lokalen Aufführungskontextes ging es ihm darum, das Oratorium als musikalische Predigt, moralische Unterweisung und Passionsandacht sowie als Zurschaustellung der oben schon genannten Pietas Austriaca zu formen. Während die Vertonung für die Uraufführung durch den Wiener Vizehofkapellmeister Antonio Caldara vornehmlich den Aufführungskontext und weniger das dichtungsästhetische Konzept Metastasios berücksichtigte, stellte die Idee des Passionsdramas für eine interkonfessionelle Adaption von La Passione wiederum die treibende Kraft dar. Harrers

24 Zu Königs Engagement für die Förderung der deutschen Sprache vgl. Günter Jäckel, "Hofpoesie und Aufklärung”, in: Geschichte der Stadt Dresden, Bd. 2: Vom Ende des Dreißigjährigen Krieges bis zur Reichsgründung, hrsg. von Reiner Groß und Uwe John, Stuttgart 2006, S. 264-278, hier S. 270.

25 Zur Pietas Austriaca vgl. Anna Coreth, "Pietas Austriaca. Wesen und Bedeutung habsburgischer Frömmigkeit in der Barockzeit", in: Mitteilungen des Österreichischen Staatsarchivs Innsbruck, Wien etc. 1954, S. 90. Vgl. auch Karl Vocelka und Lynne Heller, Die Lebenswelt der Habsburger. Kultur- und Mentalitätsgeschichte einer Familie, Graz etc. 1997, S. 14. Zur Verbindung von Frömmigkeitspraxis und Musik am Wiener Kaiserhof vgl. Friedrich Wilhelm Riedel, Kirchenmusik am Hofe Karls VI. (1711-1740). Untersuchungen zum Verhältnis von Zeremoniell und musikalischem Stil im Barockzeitalter, München etc. 1977, S. 10. 
Vertonung für die Leipziger Karfreitagsvesper mit deutscher Übersetzung erhob den dramatischen Ansatz von Metastasios Passionsdarstellung zum Prinzip der protestantischen Vergegenwärtigung der Passion. Das heißt, er machte sich die besondere dichterische Profilierung des fremdkonfessionellen Librettos zunutze, um sie in Konventionen protestantischer Passionsmusik einzubauen. Voraussetzung war, dass die Inhalte von Metastasios Passionsoratorium eine Nähe zur biblischen Passion aufwiesen. Allerdings war das Ergebnis eine recht einseitige Auslegung des „Dramatischen" der Passion, die für den Moment der Betrachtung, der sowohl in Metastasios Dichtung enthalten als auch für protestantische Passionsmusiken eigentlich essentiell war, keinen Raum hatte. Der entscheidende Punkt seiner Bearbeitung ist, dass der Erhalt des ästhetischen Kunstwerks als Ganzes Vorrang vor konfessionellen Notwendigkeiten hatte, wie sein Umgang mit dem für protestantische Passionsoratorien so wichtigen Baustein des Chorals zeigt. Anders als in protestantischen Passionsmusiken unterbrach er die Passionsdichtung nicht mit Choralstrophen, sondern positionierte diese in gebündelter Form erst am Werkende, um die Dramaturgie von La Passione zu erhalten. Die Auseinandersetzung des Thomaskantors mit Metastasios Oratorien ging über dessen Passionsoratorium hinaus. So führte Harrer 1753 seine Neuvertonungen von Metastasios La Morte d'Abel in eigener Übersetzung in der Nikolaikirche ${ }^{26}$ als auch Gioas Rè di Guida in Originalsprache im Leipziger Großen Concert auf. ${ }^{27}$

Ein Vergleich mit Schubacks Neuvertonung von La Passione für das Hamburger Konzert zeigt, wie innerkonfessionell zwischen Konzert- und Liturgiekontext unterschieden wurde: So kam Metastasios Oratorium im Hamburger Konzert "Auf dem Kamp" in der italienischen Sprache und ohne jegliche Textbearbeitungen - also so wie Harrers Gioas im Leipziger Konzert - zur Aufführung. In dieser Zeit wurden italienischsprachige Oratorienkonzerte in Hamburg häufiger ${ }^{28}$ was u. a. damit zu begründen ist, dass seit den 1740er Jahren - nach der Schließung der Hamburger Gänsemarktoper - in der Hansestadt zunehmend italienische Opern, auch Werke von Metastasio, dargeboten wurden. ${ }^{29}$ Schuback setzte mehrere Oratorien Metastasios für den neuen Hamburger Konzertsaal "Auf dem Kamp" in Musik und ließ zudem dessen Oratorien in weiteren zeitgenössischen Vertonungen aufführen. ${ }^{30}$ Die Etablierung des Hamburger Konzertoratoriums hatte bereits im frühen 18. Jahrhundert durch Telemann stattgefunden und machte die Gattung zu einer religiösen Erbauungsmusik, die von kirchlichen, inhaltlichen und stilistischen

26 Gottlob Harrer, Der Tod Abels, Partiturautograph, Leipziger Städtische Bibliotheken, Musikbibliothek: Becker III. 2. 82.

27 Gottlob Harrer, Gioas Rè di Guida, Partitur, Sächsische Landesbibliothek - Staats- und Universitätsbibliothek Dresden: Mus. 2740-D-1.

28 Vgl. Werner Menke, Das Vokalwerk Georg Philipp Telemann's. Überlieferung und Zeitfolge, Kassel 1942, S. 47ff.

29 Bärwald, Italienische Oper in Leipzig, Bd. 1 (Textteil), S. 6. Vgl. auch Josef Sittard, Geschichte des Musik- und Concertwesens in Hamburg vom 14. Jahrhundert bis auf die Gegenwart, Altona 1890, S. 76. Joachim E. Wenzel, Geschichte der Hamburger Oper 1678-1978, Braunschweig 1978, S. 29 f.

30 Bei Schubacks Vertonungen handelt es sich um La Passione (1763), La Betulia liberata (1773) und Gioas (1777). Vgl. Steffen Voss, „Die Metastasio-Vertonungen des Hamburger Syndikus Jacob Schuback. Ein Beitrag zur Geschichte des Oratoriums im Hamburg des 18. Jahrhunderts", in: Händel-Jahrbuch 45 (1999), S. 173-185, hier S. 176. 
Vorgaben weitgehend unabhängig war, sodass das ästhetische sowie gesellige Ereignis in den Vordergrund rückte. Während man in den deutschsprachigen Werken daran festhielt, die Abfolge von Rezitativ-, Arien- und Chorsätzen durch Choralstrophen zu untergliedern, beließ man die italienischen Werke in ihrer ursprünglichen Form, wie Schubacks La Passione exemplarisch zeigt. Allerdings enthält auch seine Komposition Reminiszenzen an diese Tradition, indem er im Instrumentalsatz der Sinfonia zu Beginn des zweiten Teils den Passionschoral "Wenn meine Sünd mich kränken“ zitiert (vgl. Sinfonia 19). Auf eine subtile konfessionelle Kontextualisierung des katholischen Passionsoratoriums wird also doch nicht verzichtet. Das Besondere seiner Vertonung von Metastasios Libretto besteht darin, dass er dessen Affektdarstellung empfindsam deutet. So distanziert er sich von einer traditionellen Lesart einheitlicher Affekte der Opera seria und stellt die Vielschichtigkeit der emotionalen Regungen, die in der Dichtung enthalten ist, in den Vordergrund, nämlich durch eine für Metastasio-Vertonungen unkonventionelle, aber dem zeitgenössischen Stil entsprechende flexible Handhabung der Formen Rezitativ und Arie. Schubacks Musik warb damit für eine subjektive, auf Innerlichkeit angelegte Passionsbetrachtung, die zudem nicht mehr streng konfessionell definiert war.

\section{Beispiel 3: Rezeption von Hasses I Pellegrini in Leipzig und Braunschweig}

Die Leipziger Gottesdienst-Rezeption von I Pellegrini durch Harrers Nachfolger Doles steht im Kontext der regelmäßigen Hasse-Aufführungen im Großen Concert seit den 1750er Jahren. Im Vergleich zu Metastasios La Passione war der Inhalt von I Pellegrini für eine protestantische kirchliche Aufführung weniger zur interkonfessionellen Rezeption geeignet, zum einen aufgrund des Pilgersujets - europäische Pilger sind nach Jerusalem aufgebrochen, um die Leidensstätten Jesu zu besuchen und zu verehren -, zum anderen aufgrund der zu geringen Bezugnahme auf den biblischen Passionsbericht, der für ein protestantisches Passionsoratorium zentral war. Daher wurde Hasses Musik nach dem Parodieverfahren neu textiert. Dabei erzeugte das neu geschaffene Wort-Ton-Verhältnis eine offensichtliche Spannung, die aus dem Versuch resultierte, ästhetische Elemente der Musik und konfessionelle Anforderungen an die Dichtung miteinander in Einklang zu bringen: So richtete Enderlein seine Dichtung an der Affektstruktur der Musik aus, war aber nicht darauf bedacht, den Tonfall seiner Verse an die von der Musik ausgehende Grundstimmung anzupassen. Diese war primär auf die Geschmackskonventionen des Dresdner Hofes unter Friedrich August II. und das Verständnis des Karwochenoratoriums als ästhetisch-religiöses Ereignis abgestimmt, während Enderlein im aufklärerischen Duktus die erzieherisch-moralische Komponente sowie den lutherischen Bekenntnischarakter der Passionsbetrachtung in den Vordergrund stellte. In Verbindung mit Hasses tendenziell eher unbeschwert klingender Musik wirken seine Verse befremdlich, an manchen Stellen sind Text und Musik gar unvereinbar. Die Untergliederung aller Rezitativ-Arien-Paare mit Choralstrophen macht aus Hasses Oratorium zudem ein Hybridwerk protestantischen Zuschnitts. Ein zurückgehender Blick auf Harrers Lösung der Choralfrage in La Passione verdeutlicht, dass die 
Gewichtung von Konfession und Ästhetik am gleichen Ort im gleichen Kontext - Leipziger Passionsmusik in den Hauptkirchen - differieren konnte. Dies hatte zum einen mit unterschiedlichen musikalischen Prägungen und Zielsetzungen der beiden Thomaskantoren zu tun: So war Doles durch die Kompositionsschule Johann Sebastian Bachs sowie seine vorherige Kantorentätigkeit in Freiberg kirchenmusikalisch konservativer geprägt ${ }^{31}$ als Harrer, der seine musikalische Sozialisation hauptsächlich im Umkreis des Dresdner Hofes erfahren hatte. ${ }^{32}$ Zum anderen führt diese Beobachtung zur Schlussfolgerung, dass sich das Parodieverfahren im interkonfessionellen Transfer als problematischer erwies als das der Neuvertonung, da der polyseme Charakter des Mediums Musik Grenzen hatte und nicht beliebig verwendet werden konnte, wenn es darum ging, eine neue sinnvolle Text-Musik-Einheit zu schaffen.

Die Braunschweiger Pellegrini-Rezeption setzte eigene Akzente, die das Ausmaß des interkonfessionellen Oratorienaustauschs um zwei wichtige Aspekte erweitert. Der erste Aspekt zeigt sich in Eschenburgs Übersetzung von Pallavicinos I Pellegrini. Sie war in erster Linie nicht funktional gedacht, sondern ästhetischer Natur. Der Braunschweiger Literat fokussierte den Eigenwert der italienischen Dichtung, was dem zeitgenössischen und insbesondere seinem eigenen Bestreben entsprach, den deutschsprachigen Literaturkanon mit fremdsprachlichen Werken zu erweitern. Trotzdem wurde I Pellegrini von Eschenburg mit "konfessionellen Augen" durchleuchtet, wenn er alle kritischen Stellen in Bezug auf das von Protestanten kritisierte Wallfahrtswesen durch Verse ersetzte, die das lutherische Bekenntnis stärkten. Diese Umarbeitungen waren aber wiederum so gestaltet, dass sie zu Hasses Musik passten. Hier wurde folglich nicht von einem einseitigen Interesse für Dichtung oder Musik und aus einem damit verbundenen handwerklichen Pragmatismus heraus der Versuch unternommen, ein fremdkonfessionelles Werk protestantischem Publikum zugänglich zu machen, wie es bei Enderlein der Fall war. Vielmehr ging es Eschenburg darum, sowohl die Komposition in ihrer Eigenwertigkeit als auch die Dichtung in ihrer inhaltlich-literarischen Qualität zu erhalten und dabei bestehende konfessionelle Sensibilitäten im Braunschweiger Aufführungskontext zu berücksichtigen. Das Interkonfessionelle, das durch Eschenburgs Pellegrini-Adaption entstanden war, vollzog sich folglich ausgehend von einem literarisch-intellektuellen sowie theologischen Standpunkt der Braunschweiger Dichtungskultur.

Eine anders gelagerte Pointe in diese Richtung setzte Zachariä, der Pallavicinos Wallfahrtsoratorium in seiner Neudichtung Die Pilgrime auf Golgatha - in Verbindung mit neuen dichterischen Oratorienkonventionen - als kritischen Reflex des Pilgerstoffes umarbeitete. Zudem versah er es mit freigeistkritischen Anspielungen, mit denen das Werk, ausgehend von der Pilgerthematik, eine überkonfessionelle Diskursebene zum zeitgenössischen Atheismus erreichte. Diese neue

31 Helmut Banning, Johann Friedrich Doles: Leben und Werke, Berlin 1939, S. 1-100. Andreas Glöckner, Art. „Doles, Johann Friedrich", in: Die Musik in Geschichte und Gegenwart, 2., neubearb. Ausgabe, Personenteil Bd. 5, hrsg. von Ludwig Finscher, Kassel etc. 2001, Sp. 1201-1208.

32 Ulrike Kollmar, Gottlob Harrer (1703-1755), Kapellmeister des Grafen Heinrich von Brühl am sächsisch-polnischen Hof und Thomaskantor in Leipzig, Beeskow 2006, S. 23-48. 
Dimension der Interkonfessionalität im Oratorium führte noch weiter: Albrechtsbergers Vertonung für die Wiener Tonkünstler-Societät 1781 erklang in einer Zeit, in der das Wallfahrtswesen im Kontext derjosephinischen Reformen in Misskredit fiel ${ }^{33}$ und die protestantische Pilgerkritik somit auch katholischerseits aufZustimmung stoßen konnte. Ob dieservon musikalischen Aspekten losgelöste Diskurs auf der Ebene der Oratorienlibretti von der Musik tatsächlich mitgetragen wurde, ist eine weiterführende Forschungsfrage, die an dieser Stelle nicht ausgeführt werden kann. Sie hätte die neuen Kompositionsprinzipien der Wiener Klassik, die Eigendynamik der spät aufblühenden Wiener Konzertkultur sowie die bewusste Konfessionsungebundenheit der Wiener Oratorienkonzerte in Abgrenzung zu den früheren höfischen Oratorien zu berücksichtigen.

\section{Beispiel 4: Rezeption der Oratorien Hasses in der Herrnhuter Brüdergemeine}

In den 1760er Jahren setzte in den Herrnhuter Gemeinen eine umfangreiche Rezeption von Vokal- und Instrumentalmusik nicht-brüderischer Komponisten ein. ${ }^{34} \mathrm{U}$. a. fanden Oratorien und Kantaten von Georg Friedrich Händel, Carl Heinrich Graun, Carl Philipp Emanuel Bach, Johann Heinrich Rolle, Joseph Haydn und auch Johann Adolph Hasse - das heißt Werke verschiedener konfessioneller Traditionen und Kontexte - Eingang in die brüderische Musik. Unter "Rezeption“ im Herrnhuter Kontext hat man sich allerdings etwas anderes vorzustellen als die oben beschriebenen Formen der Anverwandlung für lutherische Aufführungskontexte. Tendenziell handelte es sich in der Herrnhuter Brüdergemeine um eine extrem selektive Auswahl von Einzelsätzen - zumeist Chorsätzen - aus den Werken, die, je nachdem, wie weit Text- und Musikmaterial mit dem brüderischen Musik- und Religionsverständnis vereinbar waren, drastischen Bearbeitungen im Hinblick auf Text und Musik unterworfen wurden. Die selektive Wahl von Einzelsätzen war vornehmlich von den spezifischen, tendenziell schlichten Aufführungsbedingungen brüderischer Musik abhängig. In den musikalischen Bearbeitungen kamen die besonderen musikalisch-stilistischen Prämissen der Brüdergemeine zum Ausdruck: so etwa der Ausschluss alles "Weltförmigen“, d. h. virtuoser und tänzerischer Musik, ${ }^{35}$ dagegen die Bevorzugung gravitätischer und würdevoller Musik. ${ }^{36}$ Schließlich ging es um Deutlichkeit und in Bezug auf Vokalmusik um Textverständlichkeit, ${ }^{37}$ um ein "musiziertes Sprechen" ${ }^{\prime 38}$ zu gewährleisten. Kunstvolle Polyphonie war damit ausgeschlossen. Ferner bedeutete die Anverwandlung des fremden Repertoires in Form der Bearbeitung als solcher einen Mechanismus, die rezipierte Musik zur eigenen zu machen, ähnlich wie deutsche Sprache und Choräle bei lutherischen Bearbeitungen katholischer Oratorien.

33 Vgl. Harm Klueting, Art. „Josephinismus”, in: Religion in Geschichte und Gegenwart, Vierte, völlig neu bearbeitete Auflage, Bd. 4, hrsg. von Hans Dieter Betz et al.,Tübingen 2001, Sp. 584.

34 Walter Blankenburg, "Die Musik der Brüdergemeine in Europa”, in: Unitas Fratrum 1975, S. 378 f.

35 Wehrend, Musikanschauung, Musikpraxis, Kantatenkompositionen in der Herrnhuter Brüdergemeine, S. $114 \mathrm{f}$.

36 Ebd., S. 128.

37 Ebd., S. 125.

38 Hans-Walter Erbe, „Zur Musik in der Brüdergemeine”, in: Unitas Fratrum 1977, S. 50. 
Das Charakteristische der Herrnhuter Hasse-Rezeption besteht nun darin, dass Stimmung und Stil insbesondere der Chorsätze offensichtlich das religiöse Grundgefühl der Gemeinschaft in besonderer Weise trafen und die ästhetischen Prämissen von Hasses Komposition zu religiösen Prämissen in der Adaption umgedeutet wurden. Dies lässt sich exemplarisch anhand der vielfachen Neutextierung von Hasses Lauda aus dem oben besprochenen Oratorium I Pellegrini nachverfolgen. ${ }^{39}$ Der Begrüßungsgesang Jerusalems aus Pallavicinos Dichtung wird durch Abendmahlstexte sowie Lob- und Preisgesänge ersetzt. Der schlichte, anrührende Stil der Lauda erhält bei den Herrnhutern eine besondere andachtspsychologische Dimension. Er lässt sich als Ausdruck einer Herzensfrömmigkeit begreifen, die - befördert durch den Begründer der Gemeinschaft Graf Nikolaus Ludwig von Zinzendorf - ein wesentliches Element des brüderischen religiösen Selbstverständnisses bildete. ${ }^{40}$ Die genauen Aufführungsanlässe sind nicht dokumentiert. In Frage kommen die verschiedenen Versammlungsformen der Herrnhuter wie Liebesmahl, Singstunde, Liturgie und Kantate, in denen Musik im Zentrum stand. ${ }^{41}$

Im Vergleich zur lutherisch-protestantischen Hasse-Rezeption fällt ein fehlendes Verständnis für den ästhetischen Wert der Komposition als Ganzes auf. Indem musikalische Kategorien wie Affektkontraste wegfielen, wurde nicht nur die Idee des Oratoriums als geistliches Drama hinfällig, sondern der isolierte Satz musste in dem Moment, in welchem die innermusikalischen Strukturen des Werkes übergangen wurden, zusammenhanglos erscheinen. In der Herrnhuter Oratorienpraxis liegt damit eine extrem eigenwillige Variante interkonfessioneller Anverwandlung der Gattung im 18. Jahrhundert vor, die nicht losgelöst von der lutherischprotestantischen war, aber doch ganz individuelle Wege aufzeigte. Das verbindende Element war, dass in Hasses Musik ein Frömmigkeitsgefühl zum Ausdruck kam, das von der Idee her überkonfessionell war, weil es unabhängig von konfessionellen Dogmen und hochkirchlichen Lehrmeinungen auf religiöses Erleben, auf eine Art von religiöser Gefühlsästhetik abhob, die um und nach 1750 konfessionsübergreifend wirksam wurde. Hasses geistliche Musik verkörperte das Kirchenmusikideal schlechthin nicht nur für die zeitgenössische katholische Kirchenmusik, sondern auch für die lutherische, wie die Hasse-Rezeption der Leipziger Thomaskantoren dokumentiert, sowie für pietistische Gruppierungen wie die Herrnhuter Brüdergemeine.

39 Vgl. u. a. folgende Quellen aus dem Unitätsarchiv Herrnhut: Mus. A 13:147, Mus. A 16:209, Mus. B 30:19, Mus. B 221:1, Mus. B 221:1a, Mus. B 221:4, Mus. C 219:1, Mus. E 27:37, Mus. E 35:101, Mus. E 35:102, Mus. E 35:105, Mus. H 15:1, Mus. H 15:2, Mus. K 220:1. Vgl. auch die Quellen der Moravian Music Foundation: Johannes Herbst Collection: H 158 [2.], H 158 [2b], H 161 c 1; Salem Congregation Collection: S 115, S 220, S 326; Salem Single Sisters Collection: SS 5.4, SS 5.8.

40 Hans-Christoph Hahn, "Theologie, Apostolat und Spiritualität der evangelischen Brüdergemeine”, in: Unitas Fratrum 1975, S. 287.

41 Erbe, „Zur Musik in der Brüdergemeine”, S. 47 f.; Blankenburg, "Die Musik der Brüdergemeine in Europa”, S. 361. 


\section{Fazit und Ausblick}

Die Gründe und Impulse für den interkonfessionellen Repertoiretransfer von Oratorien im 18. Jahrhundert lagen in der Regel außerhalb konfessioneller Erwägungen, wie die in den Beispielen dargestellten Rezeptionskontexte und -modalitäten sowie die Beschaffenheit der transformierten Werke erkennen lassen. Zwar war ein gewisser inhaltlicher Konsens die Bedingung, doch die Auswahl der fremdkonfessionellen Oratorien war hauptsächlich ästhetisch motiviert. Dies dokumentiert die protestantische Rezeption der Wiener Oratoriendichtungen Metastasios und der Dresdner Oratorienvertonungen Hasses, handelte es sich hier doch um die wichtigsten Vertreter der europäischen Opern- und Oratorienkultur des 18. Jahrhunderts, die Wertschätzung über konfessionelle Grenzen hinweg genossen. Die Art und Weise, wie Oratorien neuen konfessionellen Kontexten einverleibt wurden, macht indessen sichtbar, dass je nach Aufführungsanlass - Kirche oder Konzert - in größerem oder kleinerem Umfang konfessionell bedingte textliche, musikalische und aufführungspraktische Modifizierungen und Anpassungen (Neutextierungen, Neuvertonungen, Kürzungen, Übersetzungen) notwendig waren. Dies gilt insbesondere für die Adaption der paraliturgischen höfischen Oratorien Wiens und Dresdens in protestantischen Gottesdiensten Leipzigs und Braunschweigs (vgl. Metastasios La Passione und Hasses I Pellegrini). Hier waren konfessionsspezifische Anforderungen und ästhetische Ansprüche miteinander in Einklang zu bringen, was textlich-musikalische Kompromisse zur Folge hatte. Im Hamburger, Leipziger und Braunschweiger Konzert wiederum überwog bei der Rezeption der italienisch-katholischen Werke der Kunstanspruch. Die unterschiedliche Gewichtung konfessioneller Aspekte je nach Aufführungskontext zeigt aber auch, dass diese im 18. Jahrhundert - anders als im Zeitalter der Reformation und im konfessionellen Zeitalter - weniger prinzipielle als kontextuelle Gültigkeit besaßen. Schließlich veranschaulichen die Beispiele, dass die auf Neudichtungen basierende und funktional wandelbare Musik die Frage nach der Konfessionsgebundenheit des Oratoriums im 18. Jahrhundert keineswegs eindeutig beantwortet, gerade aufgrund des vielseitig interpretierbaren Klangmediums der Musik.

Es lässt sich festhalten, dass die Konfession, gleichgültig, in welcher Weise sie jeweils relevant war - konfessionspolitisch, theologisch oder liturgisch-rituell -, weder das alleinbestimmende Merkmal der Gattung noch einen zu vernachlässigenden Faktor darstellte. Denn die höfische und städtische Musikkultur des 18. Jahrhunderts war nach wie vor konfessionell geprägt, wurde aber immer stärker von anderen, konfessionsungebundenen bzw. konfessionelle Polaritäten verdrängenden Aspekten beeinflusst: Unter anderem lösten sich die Glaubens- und Frömmigkeitspraktiken der Konfessionen in der Konfrontation mit dem Denken der Aufklärung zunehmend von einer dogmatischen Ausrichtung. Die Oratorien spiegelten diesen Wandel nicht nur wider, sondern beförderten einen konfessionsübergreifenden affektiven Zugang zu geistlichen Inhalten wie der Passion, der zugleich auf religiöse Verinnerlichung und ästhetischen Genuss zielte. Der zum Teil gemeinsame Dichtungs- und Musikstil ließ die Konfessionen trotz inhaltlicher 
und institutioneller Verschiedenheiten in ihrer religiösen Ausdrucksweise im 18. Jahrhundert näher aneinanderrücken. Das bedeutet, dass die Gattung das Spannungsfeld der Konfessionen durch gewachsene konfessionsgebundene Oratorienpraktiken nicht nur konturierte und profilierte, sondern in gewisser Weise auch überwand. Denn sie "bekannte" sich nicht nur zu einer Konfession, sondern zunehmend auch zu einer dezidierten, überkonfessionell wirksamen Art der musikreligiösen Ausdrucksweise, die im Verlauf des 18. Jahrhunderts überdies zu einer ästhetischen Verselbstständigung führen konnte.

Ein Ausblick auf die Entwicklungen im 19. Jahrhundert zeigt allerdings, dass im Zuge einer Rekonfessionalisierung im deutschsprachigen Raum, die mit nationalem Gedankengut überformt wurde ${ }^{42}$ die Oratorien eine neue Dimension des Bekenntnishaften gewannen, das sich in einer engen Verquickung von Konfessionalismus und Nationalismus manifestierte. ${ }^{43}$ Vor dem Hintergrund dieser neuen Einflussfelder des Oratoriums im 19. Jahrhundert wäre die Frage nach der Konfessionsgebundenheit oder Konfessionsungebundenheit, der interkonfessionellen und der akonfessionellen Dimensionen des Oratoriums in dieser Zeit in gleicher Weise zu untersuchen wie für die Gattungsgeschichte des 18. Jahrhunderts.

Zitation: Maryam Haiawi, „Das Oratorium im Spannungsfeld der Konfessionen: Zum interkonfessionellen Austausch von Oratorien im 18. Jahrhundert", in: Freie Beiträge zur Jahrestagung der Gesellschaft für Musikforschung 2019, hrsg. von Nina Jaeschke und Rebecca Grotjahn (= Musikwissenschaft: Aktuelle Perspektiven. Bericht über die Jahrestagung der Gesellschaft für Musikforschung 2019 in Paderborn und Detmold, Bd. 1), Detmold 2020, S. 115-129, DOI: $10.25366 / 2020.55$.

42 Olaf Blaschke, „Der ,Dämon des Konfessionalismus'. Einführende Überlegungen”, in: Konfessionen im Konflikt. Deutschland zwischen 1800 und 1970: ein zweites konfessionelles Zeitalter, hrsg. von Olaf Blaschke, Göttingen 2002, S. 13-69.

43 Vgl. dazu u. a. Linda Maria Koldau, „Nationalreligiosität und Oratorien: Bonifatius und Luther als konfessionelle Antipoden im 19. Jahrhundert", in: Musik in neuzeitlichen Konfessionskulturen (16. bis 19. Jahrhundert). Räume - Medien - Funktionen, hrsg. von Michael Fischer, Norbert Haag und Gabriele Haug-Moritz, Stuttgart 2014, S. 245-260. 


\section{Abstract}

The present study deals with interconfessional exchange of oratorios in German-speaking countries during the 18th century. In doing so, it pursues the goal of focusing on the question of the denominational or non-denominational nature of the sacred music genre, a question that has so far been insufficiently discussed in musicological and literary research. It analyses selected oratorios between 1715 and 1781 which were written at important contemporary musical locations and were received interdenominationally (Hamburg, Leipzig, Brunswick, Catholic imperial court of Vienna, Catholic Saxon court at Dresden).

The study comes to the conclusion that the oratorio of the 18th century was not defined solely by its denominational orientation, but influenced by a number of other factors reflecting the intellectual-historical upheavals of the Age of Enlightenment: contemporary musical aesthetics, socio-cultural developments (middle-class concert business), and fundamental religioushistorical dynamics that led to a distancing from dogma and to a change in piety practice.

\section{Kurzvita}

Maryam Haiawi studierte Musikwissenschaft, Kirchenmusik, Orgel und Klavier. Ihre Promotion zum Thema „Das Oratorium als konfessionelles Bekenntnis? Interkonfessioneller Austausch von Oratorien im 18. Jahrhundert" erfolgte im Rahmen einer wissenschaftlichen Mitarbeiterstelle am Graduiertenkolleg „Interkonfessionalität in der Frühen Neuzeit” der Universität Hamburg. Derzeit führt Haiawi eine Pilotstudie zur Adaption zeitgenössischer Vokal- und Instrumentalmusik in der Herrnhuter Brüdergemeine zwischen 1760 und 1850 im Kontext ihrer Musik- und Religionsauffassung durch. 


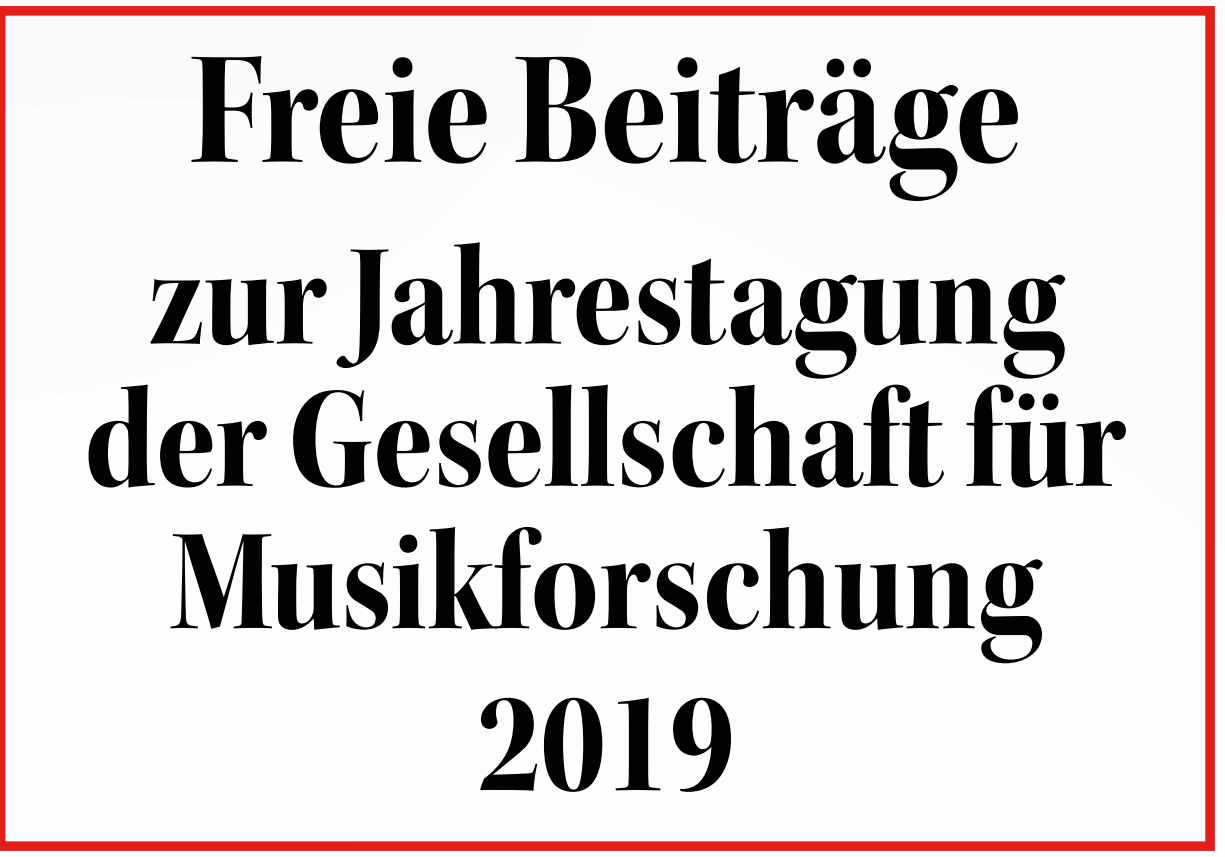

Herausgegeben von Nina Jaeschke und Rebecca Grotjahn

Musikwissenschaft: Aktuelle Perspektiven 1 
Freie Beiträge 


\section{Musikwissenschaft: Aktuelle Perspektiven}

Bericht über die Jahrestagung der Gesellschaft für Musikforschung 2019 in Paderborn und Detmold

Herausgegeben von Rebecca Grotjahn und Nina Jaeschke

Band 1 


\section{Freie Beiträge}

\section{zur Jahrestagung der Gesellschaft für Musikforschung 2019}

Herausgegeben von Nina Jaeschke und Rebecca Grotjahn

Detmold: Musikwissenschaftliches Seminar der Universität Paderborn und der Hochschule für Musik Detmold 2020 
DOI: $10.25366 / 2020.42$

Online-Version verfügbar unter der Lizenz: Urheberrecht 1.0, $<$ https://rightsstatements.org/page/InC/1.0/?language=de>

Bibliografische Information der Deutschen Nationalbibliothek

Die Deutsche Nationalbibliothek verzeichnet diese Publikation in der Deutschen Nationalbibliografie; detaillierte bibliografische Daten sind im Internet über http://dnb.d-nb.de abrufbar.

\section{Impressum}

Redaktion: Nina Jaeschke, Rebecca Grotjahn und Jonas Spieker Satz: Nina Jaeschke

(C) Musikwissenschaftliches Seminar der Universität Paderborn und der Hochschule für Musik Detmold 2020 


\section{INHALT}

Vorwort $\quad$ IX

Komponieren für das Radio: Akteure, Diskurse, Praktiken $\quad 1$

Musikwissenschaft - Feminismus - Kritik: Ein Generationenaustausch 6

\section{Stefan Alschner}

Der Wagner-Sänger Joseph Aloys Tichatschek - Vom Nachlass zum Netzwerk

\section{Alenka Barber-Kersovan}

Songs for the Goddess. Das popmusikalische Neo-Matriarchat zwischen Ethno-Beat,

erfundenen Traditionen und kommerzieller Vermarktung

Elias Berner, Julia Jaklin, Peter Provaznik, Matej Santi, Cornelia Szabó-Knotik

Musikgeschichte anders erzählen? Das Beispiel der 1970er in Österreich.

Musikhistoriographie in der Zeit der Digitalisierung

\section{Mauro Fosco Bertola}

„Ein Laut so klagevoll”. Lohengrin zwischen Richard Wagner und Salvatore Sciarrino

\section{Matthieu Cailliez}

Europäische Rezeption der Berliner Hofoper und Hofkapelle von 1842 bis 1849

\section{lacopo Cividini}

Zwischen klassischer Musikphilologie und angewandter Informatik:

Die Digitale Mozart-Edition (DME) der Stiftung Mozarteum Salzburg

\section{Marko Deisinger}

Fortschrittliche Technologie im Dienste eines Antimodernisten.

Heinrich Schenker und der österreichische Rundfunk

\section{Norbert Dubowy}

Vom Kritischen Bericht zur Kritischen Dokumentation am Beispiel der Digital-interaktiven Mozart-Edition

\section{Markus Engelhardt}

Musik zwischen Nation Building und Internationalität. Italien um 1900

\section{Maryam Haiawi}

Das Oratorium im Spannungsfeld der Konfessionen: 


\section{Judith I. Haug}

"Manch eine*r liegt, morgens noch trunken, im Rosengarten" - Rekonstruktionen

osmanischer Musikgeschichte in Gesangstextsammlungen

\section{Renate Koch}

Marcel Prawy und das erste Broadway-Musical im Österreich der Nachkriegszeit

Susanne Kogler, Julia Mair, Juliane Oberegger, Johanna Trummer

Erich Marckhl - Musikausbildung in der Steiermark nach 1945.

Brüche und Kontinuitäten

\section{Marie-Anne Kohl}

Die weinende Jury. "Geschlechtslose" Tränen bei globalen Musik-Castingshows?

\section{Fabian Kolb}

Tanztheater und filmische Ästhetik. Cineastische Einflüsse und Gestaltungsweisen in den Kompositionen für die Ballets Suédois 1920-1925

\section{Christian Lehmann}

Tempobezeichnungen von Julius Stockhausen für Die schöne Müllerin:

Ein Quellenfund

\section{Martin Link}

Signum et gens - Zur Gendersemiotik in Clara und Robert Schumanns Liederzyklus Liebesfrühling

\section{Livio Marcaletti}

„Strafspiel" und satirische Stilmittel in musikdramatischen Gattungen des frühen 18. Jahrhunderts

\section{Tobias Marx, Martin Lissner}

Thüringer Musikszene - Jugendmusikredaktionen als außerschulische musikbezogene Bildungskontexte

\section{Maho Naito}

Die Parallelität der Entstehungsprozesse der ersten beiden Symphonien Gustav

Mahlers: Instrumentation, Revision und Dirigierpraxis

\section{Elisa Novara}

Eine Schumann-Werkstatt? Zur Übertragbarkeit der Methoden vom Projekt 
Theodora Oancea, Joachim Pollmann, Jonas Spieker

Kollaborateure - Involvierte - Profiteure. Erarbeitung eines Online-Lexikons zur

Musik in der NS-Zeit

\section{Kiron Patka}

„Ich wollte eigentlich Sängerin werden." Berufsselbstbilder von Tontechniker*innen im Radio

\section{Siegwart Reichwald}

Die Leiden der jungen Clara: Das Klaviertrio Opus 17 als Ausdruck einer Neu-

Romantikerin

\section{Elisa Ringendahl}

Lied versus Oper - Pole musikalischer Gattungen bei Oscar Bie

\section{Benedikt Schubert}

Struktur und Exegese. Über Eigentümlichkeiten in der Arie "Des Vaters Stimme ließ sich hören" (BWV 7/4)

Uwe Seifert, Sebastian Klaßmann, Timo Varelmann, Nils Dahmen

Computational Thinking in der Musikwissenschaft: Jupyter Notebook als Umgebung

für Lehre und Forschung

\section{Yusuke Takamatsu}

Synthese als Modus der Prozessualität bei Schubert:

Sein spezifisches Wiederholungsprinzip im langsamen Satz

\section{Daniel Tiemeyer}

Johann Nepomuk Hummels Sonate in fis-Moll Op. 81 - Studien zu Entstehungs-

hintergrund, Rezeption und formalerStruktur

\section{Andrea van der Smissen}

Musikalische Innovation im Umfeld der Moderne und historischen Avantgarde in Ungarn

\section{Tim Ziemer, Holger Schultheis}

Psychoakustische Sonifikation zur Navigation in bildgeführter Chirurgie

\section{Magdalena Zorn}

Musik mit dem Radio hören: Über den Begriff der musikalischen Aufführung 
Gabriele Buschmeier in memoriam 


\section{Vorwort}

Die vorliegenden Bände dokumentieren die Jahrestagung der Gesellschaft für Musikforschung 2019. In den dreieinhalb Tagen vom 23. bis zum 26. September 2019 wurden in Paderborn und Detmold nicht weniger als 185 Beiträge präsentiert, verteilt auf diverse Symposien, Round tables, Freie Sektionen und Postersessions. Sie alle auf einen Nenner bringen zu wollen, ist ein Ding der Unmöglichkeit - und das ist gut so, ist es doch Ziel der Jahrestagungen, die große Vielfalt der Themen und Methoden des Faches Musikwissenschaft abzubilden. Um die thematische Vielfalt der freien Referate angemessen abbilden zu können und gleichzeitig den inhaltlichen Schwerpunkten der beiden hier publizierten Hauptsymposien ausreichend Raum bieten zu können, erscheinen diese in drei Bänden.

„Musikwissenschaft: Aktuelle Perspektiven": Der Titel der kleinen Reihe ist keine Verlegenheitslösung. Musikwissenschaft im Kontext der Digital Humanities; Musikwissenschaft und Feminismus; Musik und Medien; Musikalische Interpretation - schon die Felder, die von den vier Hauptsymposien bespielt wurden, wären noch vor wenigen Jahrzehnten allenfalls an der Peripherie das Faches zu finden gewesen. Sie entsprechen Arbeitsschwerpunkten der Lehrenden am Musikwissenschaftlichen Seminar der Universität Paderborn und der Hochschule für Musik Detmold, das die Tagung ausrichtete. Zugleich nehmen sie Bezug auf aktuelle Ereignisse und Entwicklungen. So erwuchs das von Andreas Münzmay und Joachim Veit organisierte Symposium „Brückenschläge - Informatik und Musikwissenschaft im Dialog" unmittelbar aus den Erfahrungen im Virtuellen Forschungsverbund Edirom (ViFE) und im fakultäten- und hochschulübergreifenden Zentrum Musik-Edition-Medien (ZenMEM). Der 200. Geburtstag von Clara Wieck/Schumann war der Anlass für das von Rebecca Grotjahn geleitete Symposium „Die Begleiterin - Clara Schumann, Lied und Liedinterpretation", das in enger Kooperation mit der Hochschule für Musik Detmold durchgeführt wurde. Das Hauptsymposium „Brückenschläge" wird in einem separaten Band publiziert (Bd. 3 der vorliegenden Reihe). Im Rahmen dieses Symposiums führte die von Stefanie Acquavella-Rauch geleitete Fachgruppe Digitale Musikwissenschaft eine Posterpräsentation durch, die von den Beiträger*innen erfreulicherweise zu kürzeren Texten umgearbeitet wurden, sodass sie hier ebenfalls, zusammen mit den Postern,

publiziert werden können. Hinzu kommen einige Beiträge, die bereits bei der Jahrestagung 2018 in Osnabrück präsentiert wurden. Auch das Hauptsymposium "Die Begleiterin" wird in einem eigenen Band (Bd. 2) publiziert. Die Beiträge zu den beiden anderen Hauptsymposien hingegen werden an anderen Orten veröffentlicht; in Band 1 („Freie Beiträge zur Jahrestagung der Gesellschaft für Musikforschung 2019") der vorliegenden Publikation finden sich jedoch Einführungen und Abstracts. Das Symposium „Komponieren für das Radio" unter Leitung von Antje Tumat und Camilla Bork (Katholieke Universiteit Leuven) behandelte Einflüsse des Mediums auf Kompositionsprozesse sowie durch radiophone Kompositionen bzw. radiophonen Klang ausgelöste Diskurse. Sarah Schauberger und Cornelia Bartsch (Universität Oldenburg) nahmen das 25-jährige Jubiläum der Fachgruppe Frauen- und Genderstudien zum Anlass für einen Generationenaustausch zum Thema "Musikwissenschaft - Feminismus - Kritik": Was wa- 
ren vor einem Vierteljahrhundert Inhalte und Aufgaben einer feministischen Musikwissenschaft und wie kann sich diese heute positionieren?

Bewusst haben wir im Tagungsbericht auf inhaltliche Eingriffe in die Beiträge verzichtet. ${ }^{1}$ Das gilt besonders für die Freien Referate: Es galt, den Charakter der Jahrestagung als Forum für ,freie', d. h. innovative und auch experimentelle Gedanken zu wahren. Einige Kolleg*innen, die die Tagung mit Vorträgen und Posterpräsentationen bereichert hatten, haben sich gegen eine Publikation im vorliegenden Band entschieden - sei es, weil sie eine Möglichkeit fanden, ihre Beiträge in einem inhaltlich passenderen Rahmen zu veröffentlichen, sei es, weil ihre Überlegungen in ihre entstehenden Qualifikationsschriften fließen sollen, oder sei es, weil sie von den Autor*innen in der vorgetragenen Form zunächst verworfen wurden. Auch damit erfüllt eine Freie-Referate-Sektion ihren Zweck: Die Diskussionen mit der versammelten Fach-Öffentlichkeit sollen dabei helfen, Gedanken weiterzuentwickeln und zu verändern. In diesem Sinne sei allen Beteiligten - den Autor*innen, den nichtpublizierenden Referent*innen und den Mit-Diskutant*innen - ganz herzlich gedankt für ihr Mitwirken bei der Tagung.

Unser herzlicher Dank gilt einer Reihe weiterer Personen, die zum Gelingen dieser drei Bände beigetragen haben. Hier ist besonders Jonas Spieker zu nennen, der uns tatkräftig bei der Redaktion geholfen hat. Andrea Hammes (SLUB Dresden) sei herzlich für die Aufnahme unseres Bandes auf musiconn.publish gedankt - wir freuen uns, damit unsererseits zur Etablierung dieser innovativen Publikationsplattform beizutragen.

Erneut möchten wir an dieser Stelle allen Menschen danken, die uns bei der Organisation, Ausrichtung und Finanzierung der Tagung selbst unterstützt haben: der Präsidentin der Universität Paderborn, Prof. Dr. Birgitt Riegraf, dem Rektor der Hochschule für Musik Detmold, Prof. Dr. Thomas Grosse, den Kolleginnen und Kollegen der beiden beteiligten Hochschulen, dem Vorstand der Gesellschaft für Musikforschung, der Universitätsgesellschaft Paderborn und allen Sponsoren. Besonders dankbar sind wir den Mitarbeiter*innen und den studentischen bzw. wissenschaftlichen Hilfskräften des Musikwissenschaftlichen Seminars, die bei der Vorbereitung und Ausrichtung der Tagung immensen Einsatz zeigten - stellvertretend sei an dieser Stelle Johanna Imm erwähnt, die zusammen mit Nina Jaeschke das Herz des Organisationsteams bildete.

Wir widmen diese Reihe Dr. Gabriele Buschmeier, dem langjährigen Vorstandsmitglied der Gesellschaft für Musikforschung, die kurz vor der Publikation dieses Bandes unerwartet verstarb.

Detmold, im September 2020

Rebecca Grotjahn und Nina Jaeschke

Zitation: Rebecca Grotjahn und Nina Jaeschke, „Vorwort”, in: Freie Beiträge zur Jahrestagung der Gesellschaft für Musikforschung 2019, hrsg. von Nina Jaeschke und Rebecca Grotjahn (= Musikwissenschaft: Aktuelle Perspektiven. Bericht über die Jahrestagung der Gesellschaft für Musikforschung 2019 in Paderborn und Detmold, Bd. 1), Detmold 2020, S. IX-X, DOI: 10.25366/2020.43.

1 Freigestellt war den Autor*innen auch, ob sie sich für eine gendersensible Sprache entscheiden bzw. welche Form des Genderns sie bevorzugen. 